\title{
Propagation of a \\ Liquid-Liquid Explosion
}

\author{
Francis H. Harlow \\ Hans M. Ruppel
}

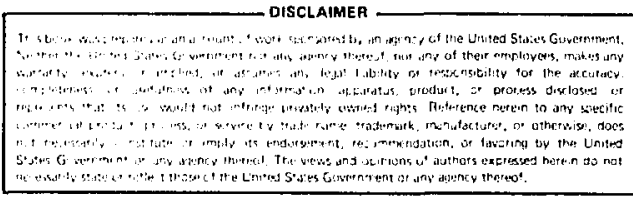

L.(9) A A. 
PROPAGATION OF A LIQUID-LIQUID EXPLOSION

by

Francis H. Harlow and Hans M. Ruppel

\begin{abstract}
Direct contact between two liquids, one cold and the other hot, may be precluded by the preserse of a vapor film. Bridging of this film by one or both flids results in rapid local boiling, which may initiate a propagating liquid-ilquid explosion. We discuss a mechanism for the propagation that involves implosion of the film, rapid mixing of the fluids, heat exchange to warn the cold fluid above the temperature for spontaneous nucleation, and the explosive generation of vapor, which in turn continues to sustain the film implosion. Plausibility for the model is demonstrated by means of numerical studies by high-speed computer.
\end{abstract}

\title{
I. INTRODUCTION
}

When hot material is brought into contact with a liquid, the latter may boil in any of several ways. The most peaceful occurs when the matertal is very hot so that a vapor film is formed, serving as an insulative blanket that greatly impedes heat transfer. The most violent can occur when the hot material is also a fluid; it is called a liquid-liquid explosion.

This violeut process is characterized by extemely rapid boiling in a confined volume. ' Evidence supports the idea that two features are required. One is rapid mixing of the two liquids so as to achieve a large interfacial area for heat transfer. The other is persistence of liquid-liquid contact for long enough to heat large quantities of the colder fluid above its bolling temperature, to the point of spontaneous nucleation.

A variety of mechanisms has been proposed to explain how these features can occur. That they do, indeed, occur is amply demonstrated by the observations of numerous investigators. Some examples of liquid combinations known to be capable of producing liquid-liquid explosions include 
1. molten tin or aluminum with water, ${ }^{1}$

2. water with liquified natural gas, ${ }^{2}$

3. molten uranium oxide with molten sodium, ${ }^{3}$ and

4. molten lava with sea water. ${ }^{4}$

In each case the first liquid is the source of heat and the second bofls to generate the high-pressure vapor.

There are two principal questions to be considered concerning the material dynamics in a liquid-liquid explosion. First, how is the explosion initiated? Second, how does it propagate?

Initiation can be either induced or spontaneous. Induction can be accomplished by some process that collapses the film and brings the liquids into direct contact. Droplets of hot fluid injected into the cold fluid, for example, have been exploded by means of a compression pulse. ${ }^{5}$ Collapse of the film on one side of the droplet is followed by a localized burst of vapor that sends fragments of the droplet into direct contact with cold liquid.

Spontaneous Initiation can occur whenever the appropriate contact between fluids is produced by a natural (nonintentiona1) fluctuation.

Propagation of a liquid-liquid explosion can occur in several different ways. Propagation through a dispersed field of droplets is a topic that has received considerable investigation ${ }^{5}$ and will not be discussed further in this report. Our concern is with the propagation along an interface between the two fluids. For a small droplet of one fluid in the other, collapse of the film over a localized area can produce a small inttiating explosion that is sufficiently disruptive to accomplish a rapid close nixing of all the available fluid in the droplet, so that propagation is not an 1ssue. It is for the case of a large drop or, at the extreme, a plane interface between the two fluids, that the mechanism for propagation is of current concern.

Ochiai and Bankoff ${ }^{6}$ visualize interface propagation in terms of cratering in each fluid as a result of a aicroexplosion from the infitiating contact. The lateral splash from each crater throws droplets across the filn gap. If these move wth sufficlent speed, described in terns of a critical impact Weber number, then the splashing drops can maintain sufficient contact for the praduction of second-generation microexplosions. These in turn generate further lateral splashes and the process continues.

The experimental results of Board and Hall ${ }^{l}$ suggest a sowewhat different nechanisw for propagation. The essential features are 1llustrated in Fig. 1. 2 
The traveling-wave configuration moves to the right with esse- ially constant speed. C denotes the region of very rapid boiling, where superheated cold liquid boils to form vapor at high density, temperature, and pressure. Thus, $C$ is a moving region with a continuous source of gas emitting pressure waves. The pressure supports shocks $S_{1}$ and $S_{2}$, both of which propagate through the liquids at speeds much greater than the shock speed in the film. (Typical liquid sound speeds for materials of relevance are three to four times greater than the sound speeds in a typical vapor.) Thus the shocks implode the film, deflecting the interfaces inwards, preceded by transmitted shocks $S_{1}^{\prime}$ and $S_{2}^{\prime}$. Until the transmitted shocks reach the opposite surfaces, point $A$, the imploding interfaces are stable. From $A$ to $B$, the reflecting shocks decelerate the interfaces, which at this stage are unstable in the Rayleigh-Taylor sense. With ample perturbation from initial irregularities, the surface waves grow rapidly in amplitude. Indeed the tips of the "spikes" or "fingers" free fall almost as though there were no deceleration, and are plunged with great velocity into contact and interpenetration between the two fluids. Heat transfer takes place between $B$ and $C$, until the superheated cold liquid reaches the temperature of spontaneous nucleation. Rapidly expanding vapor drives the fluid apart, region $D$, and emits the continuous pressure replenishment to shocks $S_{1}$ and $s_{2}$.

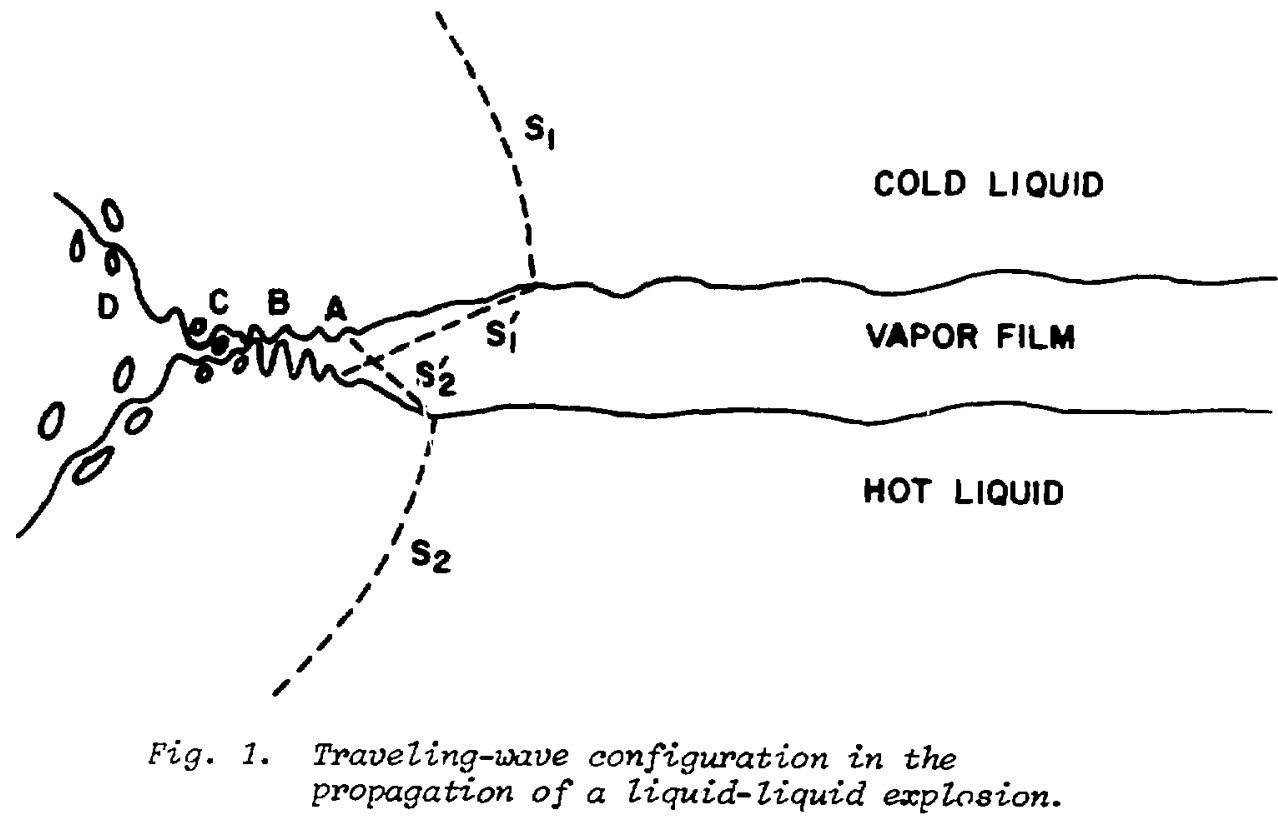


To verify the proposed mechanism for propagation will require sophisticated experimental investigations and careful theoretical analyses. This report describes some progress in the latter direction.

A full theoretical study will combine single-phase and multiphase flow dynamics, heat transport, and phase transitions. The necessary equations will require a time-varying resolution in at least two space dimensions, and will be amenable to solution only by means of numerical approximations and high-speed computers. At this stage, we have incorporated only part of the necessary physics into our computer studies, and accordingly have demorstrated plausibility for the proposed mode1, but have not yet proved its validity.

\section{SPECIFICS OF THE MODEL}

Consider the two liquids to be in horizontal stratification, the one with lesser density lying above the vapor film that separates them. Their initial temperatures are $T_{1}$ and $T_{2}$, and these temperatures tend to persist far from the film-liquid interfaces. Let the boiling-point (saturation) temperature of No. 1 be $T_{1 b}$ and suppose that

$$
\mathrm{T}_{1} \leqslant \mathrm{~T}_{1 \mathrm{~b}} \ll \mathrm{T}_{2}
$$

In addition, the freezing-point temperature of No. $2, T_{2 f}$, may exceed $T$, but whether it is essential for the occurrence of liquid-liquid explosions that $T_{1 b}<T_{2 f}$ remains to be deteriained.

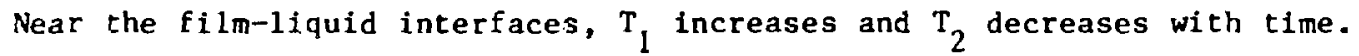
As long as $T_{2}$ is sufficiently greater than $T_{1 b}$, then liquid No. $I$ continues to boil fast enough to maintain the vapor film between the two liquids (the film boiling regime). Whichever liquid lies above the film will be penterated by bubbles of vapor, while fingers of the upper liquid will tend to fall through the film in the usual manner of Rayleigh-Taylor instability.

In fully developed film boiling, the fingers never touch the lower liquid. Whether the colder liquid is above or below the filn, the fingers that would otherwise span the film gap are impeded by the rapid production of vapor, which increases as a result of enhancenent of heat flux where the fluids approach close proximity. Except for the relatively sluggish notion of the bubbles, fully developed fila botling is a peaceful process. 
As $\mathrm{T}_{2}$ decreases near the surface, the flux of heat across the film also decreases, ultimately to the extent that fully developed film boiling cannot be maintained. At this stage, fingers of the upper fluid occasionally touch the lower fluid and the regime is called transition boiling. Although the vapor film is a good insulator against rapid heat transport, the direct contact between fluids enables the very rapid conduction of heat between them. As a result, part of the colder fluid can be superheated to the temperature of spontaneous nucleation, then boll with sudden violence, producing a burst of vapor from liquid No. 1. We ca!l this a microexplosion.

As $T_{2}$ decreases still further, the frequency of microexplosions increases, and the average heat flux across the film gap is further enhanced. The relative quiescence of fully developed film boiling has been replaced by the violent dynamics induced by direct liquid-liquid contact.

A consequence of each microexplosion is the cratering of fluid on buth sides of the $\mathrm{film}$ and the generation of splash on the periphery of each crater. Splashed liquid on each side of the crater is thrown rapidly through the film, with droplets of both fluids encountering each other. The result is a ring of microexplosions, which in turn induces a torus of cratering and lateral splash. It has been suggested that repetition of this process could propagate the sequence of microexplosions across the entire interface, resulting in a macroexplosion. 6

The properties of this propagation model have not yet been explored in detail. To describe the observed violence of liquid-liquid explosions, a valid model must predict very rapid propagation, which is difficult to visualize as a result of multiple splashes.

Single and/or multiple splash effects, however, very likely relate directly to the initiation of a large-scale liquid-liquid explosion. Localized rapid pressure generation would tend to implode the adjacent film. All that is required for the buildup of a propagating explosion would be the production of at least as much vaporization pressure as resulted from the initiating splashes.

Once the explosion has commenced to propagate across a plane interface, we expect that the configuration will move as an essentially steady-state traveling wave, as shown in Fig. 1. In this mode 1, the total effect of the explosion would not depend on the thickness of each liquid above some critical dimension because only limited volunes of the materials can be mixed, and rapid heat exchange to fluid far from the ircerface would not occur. Nevertheless, the effects of a 
macroexplosion could be dramatic, although not to the extent predicted by the assumption of rapid heat exchange throughout the bulk of both fluids. If the upper fluid were a thin layer over the lower one (for example, 11quified natural gas spread over water) then the consumption of tile entire upper fluid can be imagined in a single liquid-liquid macroexplosion. Thick layers of molten magma under sea water, however, would be precluded in this model from the realization of full explosive potentiality in a single event, although a periodic sequence of explosions could be expected as the back-flow of water re-established the necessary conditions for each new initiation and propagation.

Quenching of propagation cuuld be expected to limit the intensity of a particular explosion. A tendency to quench might result from spatial variations in film thickness, in the thickness of one or both liquids, or in boiling regime due to temperature and/or other inhomogeneities.

Some of the essential features of a computer code for the full analysis of this type of liquid-liquid explosion are the following.

1. Time varying resolution in two (and ultimately three) space dimensions.

2. Capability for large distortions and interpenetration of three materials, the cold and hot liquids and the film.

3. Flow speeds varying from far subsonic to Mach numbers near unity.

4. Heat transport through the materials from both convection and conduction.

5. Boiling, subject to the condition of previous nucleation or circumstances allowing for spontaneous nucleation.

To permit calculations with all these features requires a technique like the Particle-in-Cell (PIC) ${ }^{7}$ method, as modified for multiphase flow circumstances. ${ }^{8}$

The present study has ignored many of these complicating considerations. Our goal at this stage has been to demonstrate film implosion in a traveling wave, as a basis for showing the plausibility of mixing, heat transfer, and continuous explosive boiling in the region downstream from the implosion. To investigate the full problem will require the extension of existing numerical solution rechniques, rather than the development of wholly new procedures.

$\therefore i$. THE NUMERICAL STUDY

The computer code, SALE-2D, ${ }^{9}$ has been modified to calculate the dynamics of $\because \because \quad:$ iferent fluids. Our plausibility study bas applied this code to the dynan$\therefore s: z, \lambda c$ liquid separated into two regions by a layer of film. The configura- 
Eion is shown in Fig. 2a. The dashed line is a plane of symmetry. Fig. 2b 11lustrates the upper half, which is resolved by the calculation. Liquid and vapor are input through the right boundary at the liquid sound speed, establishing a coordinate system that travels with the propagating explosion wave. The dashed line in Fig. $2 b$ is an arbitrarily specified region in which the liqufd boils to form additional vapor, with a phase transition rate that is directly proportional to the volume fraction of liquid. The left boundary can be maintained at an elevated pressure corresponding to the expected temperature attained during constant-volume boiling after mixing with the hot fluid.

For the calculation, the entire region is subdivided into computational cells, and the time-varying dynamics are developed from an initial configuration by the numerical integration of finite-difference approximations to the full nonlinear equations of motion. Details are presented in Ref. 9.
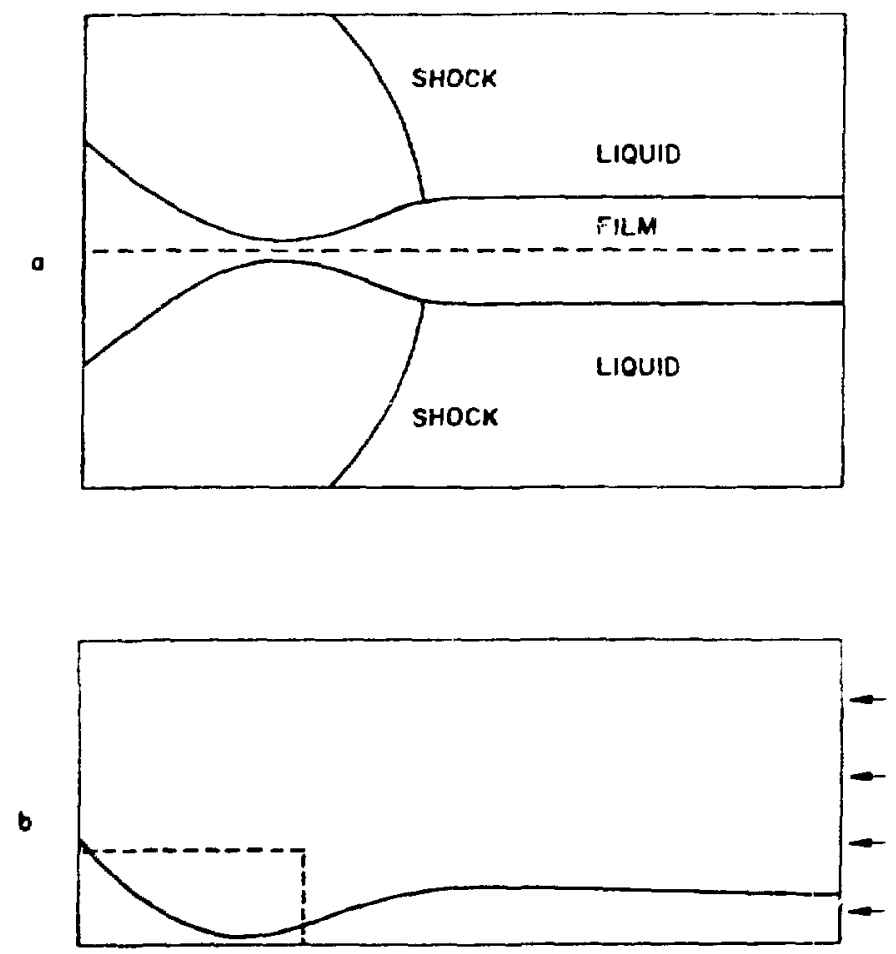

Fig. 2. Configuration for tive SiLE-2D calaulatione. 
Typical initial conditions have liquid and an unimploded film of uniform thickness all moving to the left with the same velocity (the incoming liquid sound speed). The calculation proceeds until a steady state is achieved.

A crucial feature for the attainment of steady state is strong momentum coupling between the liquid and vapor. Before implosion, this coupling ts not felt. After implosion, where the liquid and vapor mix as a result of the (unresolved) interface instability, the couplirg is essential for carrying the vapor through the boiling region. The detailed modeling of this momentum transfer has been discussed elsewhere; ${ }^{10}$ here we accomplish it by a very simple sharing term in the momentum equation. Calculations without this coupling exhibit upstream propagation of a shocked region through the $f i l m$, slowing the vapor to rest. Physically reasonable levele of coupling, however, show the expected film carrythrough, which is required for the attainment of steady-state propagation.

Typical results are illustrated in Figs. 3-6 at a tine aftar the flow configuration is close to steady state.

The volume fraction of vapor in fip. 3 varies from 1.0 at the rentrance on the lower right, to n.0 in the pure fluid above the vapor laver. The contours with $H$ have volume fraction 0.87 , and the contour intorval is 0.1 ,.

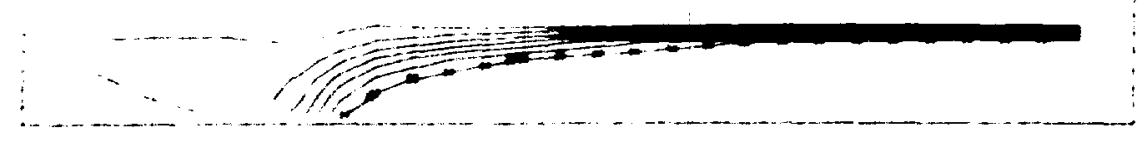

Fig. z. Contours of varor volume fraction. 


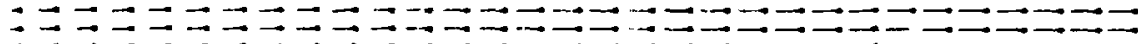

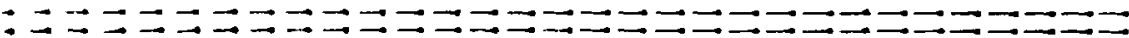

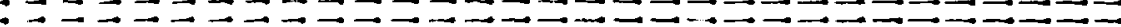

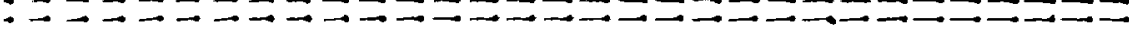

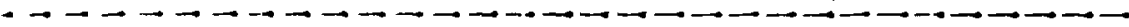

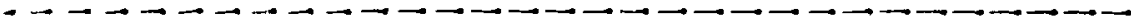

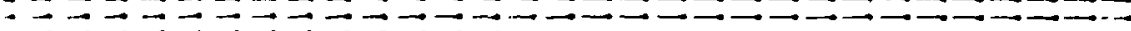

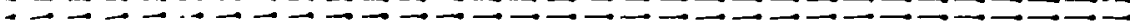

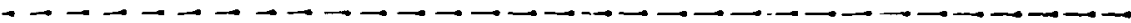

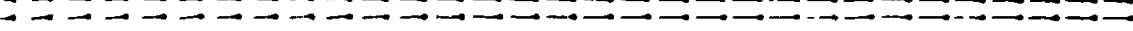

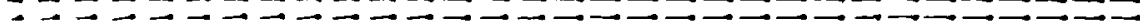

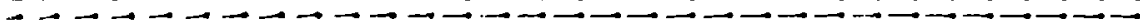

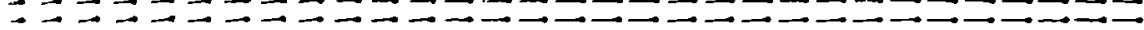

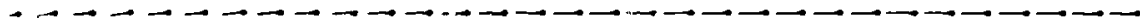

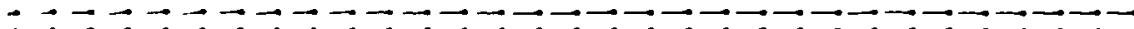

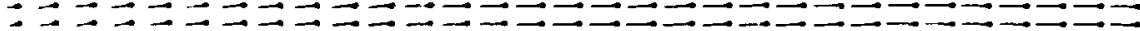

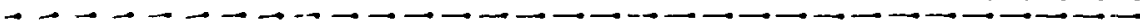

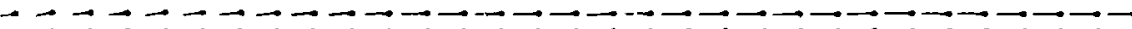

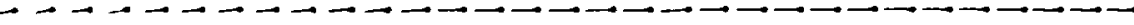

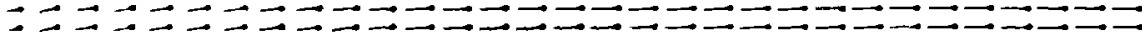

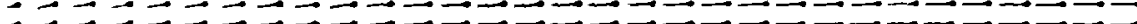

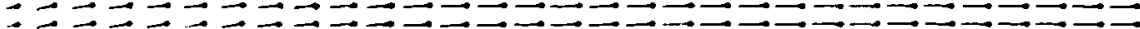

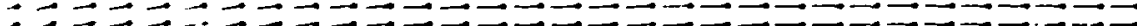

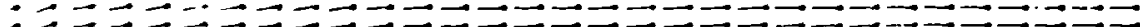

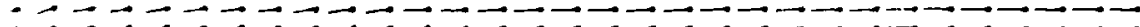

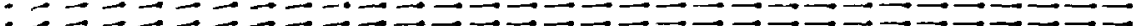

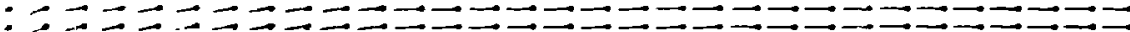

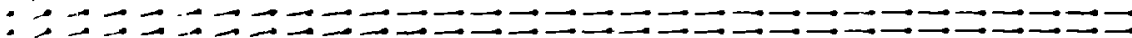

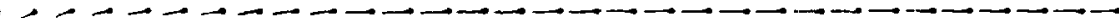

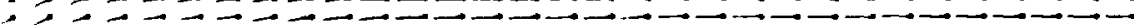

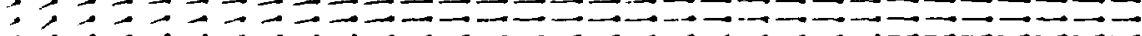

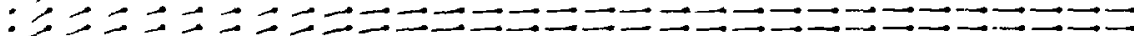
-

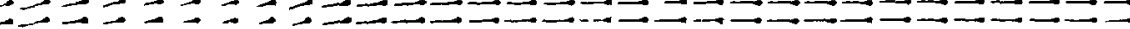

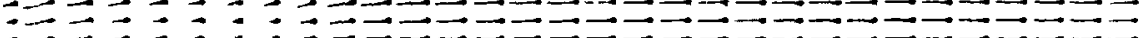

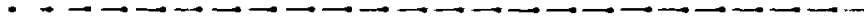

Fig. 4. Velocizi vecturs.

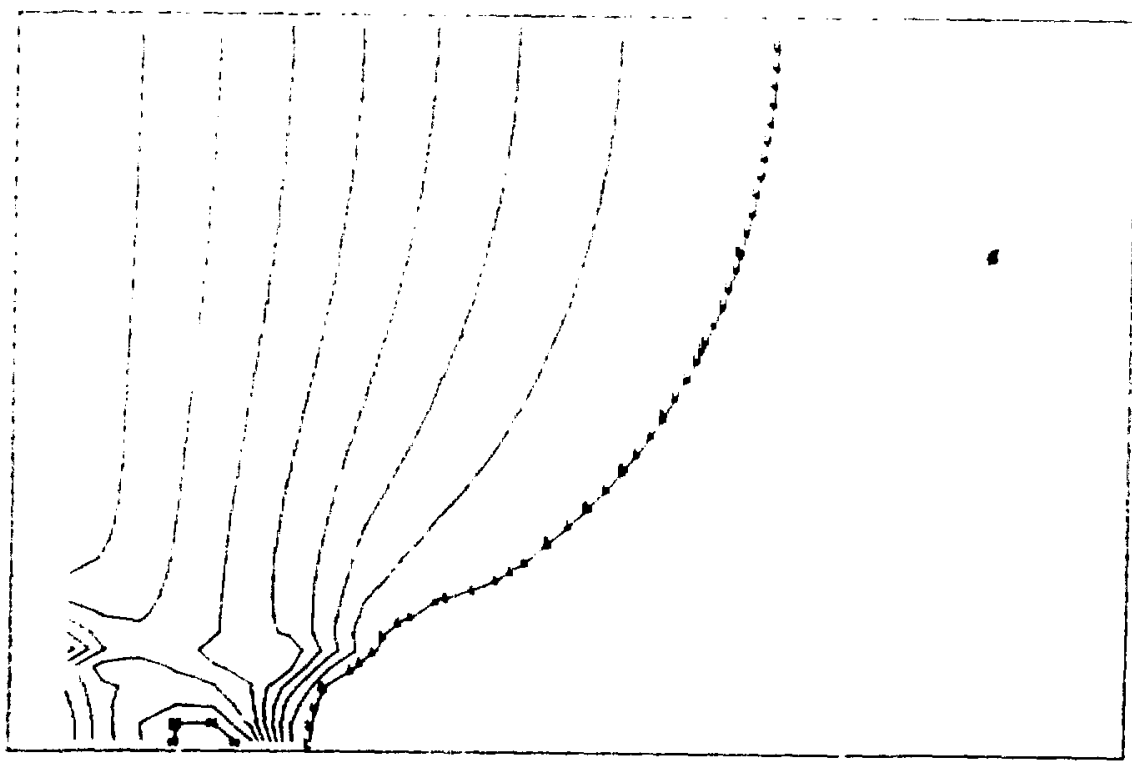

Fig. 5. Pressure contours. 


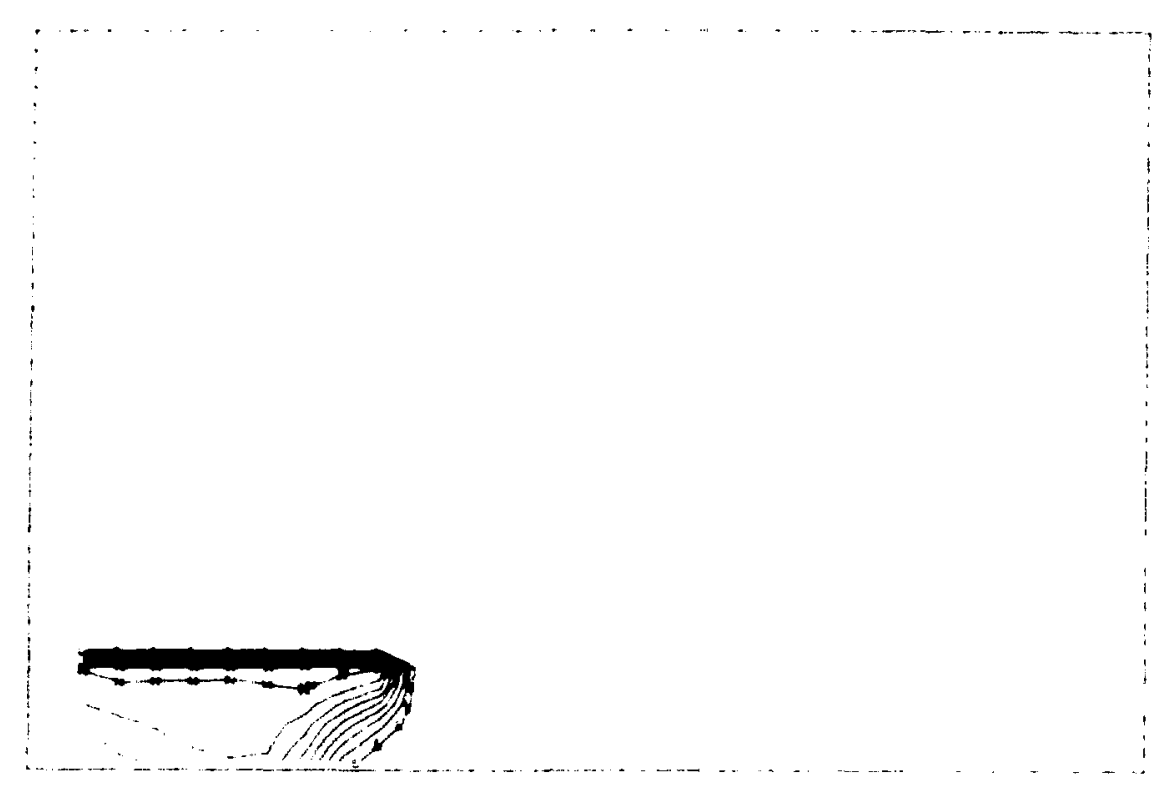

Fig. 6. Contours of boiling rate.

Figure 4 shows the velocity vectors for the motion of both fluid and vapor. The maximun speed in the system is $117 \mathrm{~cm} / \mathrm{ms}$, which is slightly greater than the incoming material speed and sound speed in the fluid, chosen to be $110 \mathrm{~cm} / \mathrm{ms}$. The vapor-fluid mixture is nearly stagnant near the point of film convergence; at later times in the calculation the speed at that point increases slightly and the vapor flow never chokes.

Figure 5 shows the contours of pressure, varying from a high in the vicinity of corvergence to a low further upstream in both materials. The II contour corresponds to 3328 bars and the low is 333 bars.

Contours of boiling rate are illustrated in Fig. 6, with $H$ corresponding to $90 \mathrm{gm} / \mathrm{cm}^{3} / \mathrm{ms}$ and $\mathrm{L}$ to $10 \mathrm{gm} / \mathrm{cm}^{3} / \mathrm{ms}$.

IV. DISCUSSION

The results presented here emphasize the preliminary nature of our investigation and cannot be cited as conclusive proof for the propagation model. They show, however, that the model is plausible and merits further investigation, using computer codes with much more of the physics carefully included. We also 
suggest that experiments be performed to search for evidence by which to verify or contradict the propagation model.

\section{REFERENCES}

1. S. J. Board and R. N. Hall, "Thermal Explosions at Molten TIn/Water Interfaces," in Moving Boundary Problems in Heat Flow and Diffusion (edited by J. R. Ockendon and W. R. Hodgkins) pp. 259-269, Oxford University Press, 1975.

2. R. P. Koopman and C. D. Lind, "Experimental Plan for $40-m^{3}$ Liquified Natural Gas (LNG) Dispersion Tests, Suppl. l, 1981 Tests," Lawrence Livermore Laboratory report UCID-18585, Suppl, I (April 1981).

3. Proc. Fourth CNSI Meeting on Fuel Coolant Interactions in Nuclear Reactor Safety, Bournemouth, United Kingdom, April 2-5, 1979. CNSI Rept. 37, 3 Vols., October 1979, R. B. Tattersal1, Ed. (CNSI; OECD Nuclear Energy Agency; 38, Boulevard Sarchet; 75016 Paris, France).

4. Stirling A. Colgate, "Dynamic Mixing of Water and Lava," Nature 244, 552 (1973).

5. M. L. Corradini, "Analysis and Modelling of Steam Explosion Experiments," Sandia National Laboratories report No. SAND80-2131 (1981).

6. M. Ochiai and S. G. Bankoff, "Liquid-Liquid Contact in Vapor Explosions," Proc. of the Intern. Meeting on Fast Reactor Safety (USERDA) Conf. No. 761001 , p. 1843 , Oct. 5, 1976.

7. F. H. Harlow, "The Particle-in-Cell Computing Method for Fluid Dynamics," Methods in Comput. Phys. 3, 319-343, Academic Press (1964).

8. T. L. Cook, R. B. Demuth, and F. H. Harlow, "Multiphase Interpretation of Shocked Materials," Los Alamos National Laboratory report No. LA-7578 (1979); "PIC Calculations of Mulitphase Flow," J. Comput. Phys. 41, 51 $(1981)$.

9. A. A. Ainsden, 4. M. Ruppe1, and C. W.: Hirt, "SALE: A Simplified ALE Computer Program for Fluid Flow at All Speeds," Los Alamos Scientific Laboratory report No. Lh-8095 (June 1980).

10. B. J. Daly and F. H. Harlow, "Scaling and Constitutive Relationships in Downcomer Yodeling," Los Alamos Scientific Laboratory report No. 7610 (February 1979). 